\title{
Development of sesbania mosaic virus nanoparticles for imaging
}

\author{
G. P. Vishnu Vardhan ${ }^{1} \cdot$ M. Hema ${ }^{3}$. C. Sushmitha ${ }^{1} \cdot$ H. S. Savithri ${ }^{1}$. $\cdot$ Usha Natraj $^{1} \cdot$ M. R. N. Murthy ${ }^{2}$
}

Received: 6 August 2018 / Accepted: 22 October 2018 / Published online: 14 November 2018

(c) Springer-Verlag GmbH Austria, part of Springer Nature 2018

\begin{abstract}
The capsids of viruses have a high degree of symmetry. Therefore, virus nanoparticles (VNPs) can be programmed to display many imaging agents precisely. Plant VNPs are biocompatible, biodegradable and non-infectious to mammals. We have carried out bioconjugation of sesbania mosaic virus (SeMV), a well characterized plant virus, with fluorophores using reactive lysine-N-hydroxysuccinimide ester and cysteine-maleimide chemistries. Monitoring of cellular internalization of labelled SeMV nanoparticles (NPs) by confocal microscopy and flow cytometry showed that the particles have a natural preference for entry into MDA-MB-231 (breast cancer) cells, although they could also enter various other cell lines. The fluorescence of SeMV NPs labelled via the cysteines with Cy5.5 dye was found to be more stable and was detectable with greater sensitivity than that of particles labelled via the lysines with Alexa Fluor. Live-cell imaging using SeMV internally labelled with Cy5.5 showed that it could bind to MDA-MB-231 cells in less than 5 minutes and enter the cells within 15 minutes. The particles undergo endolysosomal degradation by $6 \mathrm{~h}$ as evidenced by their co-localization with LAMP-1. Far-western blot analysis with a HeLa cell membrane protein fraction showed that SeMV interacts with 54-, 35- and 33-kDa proteins, which were identified by mass spectrometry as vimentin, voltage-dependent anion-selective channel protein (VDAC1), and annexin A2 isoform 2 (ANXA2), respectively, suggesting that the particles may bind and enter the cell through these proteins. The results presented here demonstrate that the SeMV NPs provide a new platform technology that could be used to develop in vivo imaging and targeted drug delivery agents for cancer diagnosis and therapy.
\end{abstract}

\section{Introduction}

Viral nanoparticles (VNPs) are naturally occurring, highly symmetrical, self-assembled molecular structures that can be developed as excellent platforms for various biotechnological applications [14]. VNPs do not normally have the intrinsic

Handling Editor: Stephen John Wylie.

Electronic supplementary material The online version of this article (https://doi.org/10.1007/s00705-018-4097-y) contains supplementary material, which is available to authorized users.

M. Hema

hemamasarapu70@gmail.com

$\triangle$ H. S. Savithri

hss@alumni.iisc.ac.in

1 Department of Biochemistry, Indian Institute of Science, Bengaluru, India

2 Molecular Biophysics Unit, Indian Institute of Science, Bengaluru, India

3 Department of Virology, Sri Venkateswara University, Tirupati, India ability to carry drugs, imaging agents, targeting ligands, or other molecular moieties. However, the desired ligands can be attached to internal, external, and intersubunit residues of VNPs by direct chemical conjugation [19] and/or by genetic engineering techniques $[11,15]$. Plant VNPs are particularly useful for biomedical applications because of their non-infectious, non-hazardous, and biodegradable nature, low cost of production, and biocompatibility [7]. Plant VNPs based on cowpea mosaic virus (CPMV), cowpea chlorotic mottle virus (CCMV), brome mosaic virus (BMV), tobacco mosaic virus (TMV) and potato virus X (PVX) and virus-like particles (VLPs) of sesbania mosaic virus (SeMV) and physalis mottle virus (PhMV) are being explored for various biomedical applications $[6,16,17,22,24]$. Thus, each plant VNP is unique in its size, shape and charge distribution on the surface, which in turn influences the in vivo behaviour and fate of modified VNPs. Therefore, there is potential for development of new plant VNP-based platforms for biomedical applications [12]. In the present study, due to its robustness and stability, ease of production in high yields, and availability of a well-characterized structure, sesbania mosaic virus (SeMV) nanoparticles 
were used for developing a platform for in vitro imaging applications.

SeMV belongs to the genus Sobemovirus and infects Sesbania grandiflora plants. Virus particles of $30 \mathrm{~nm}$ diameter consist of a capsid constructed from 180 coat protein (CP) subunits that encapsidates a single-stranded positive-sense monopartite RNA genome of $4.1 \mathrm{~kb}$. The icosahedral asymmetric unit consists of three subunits termed A, B and C that have slightly different conformations. The type A subunits form 12 pentamers, while the $\mathrm{B}$ and $\mathrm{C}$ subunits form 20 hexamers, resulting in $\mathrm{T}=3$ capsids [4]. The complete genome sequence of SeMV has been determined [13], and a full-length infectious clone has been generated [8]. SeMV can be easily purified in large quantities, and the purified virus particles are extremely stable with a $\mathrm{T}_{\mathrm{m}}$ of $91^{\circ} \mathrm{C}$ and do not undergo denaturation even at $6 \mathrm{M}$ guanidine hydrochloride (GuHCl) [20]. Detailed studies have been carried out on SeMV assembly, replication, movement and the structure-function relationships of the viral proteins [3]. Biodistribution and toxicity studies of SeMV NPs in mice have shown that they are safe and nontoxic [21]. We have shown earlier that the coat protein (CP) of SeMV readily assembles into VLPs when expressed in E. coli and can tolerate large insertions of 50-70 amino acids at the $\mathrm{N}$ - and C-termini and in the loop region without affecting the assembly of VLPs, thus demonstrating that SeMV is a good candidate for developing various platforms for biomedical applications [2]. It has been demonstrated that insertion of the B domain of Staphylococcus protein A into a surface-exposed loop of the SeMV CP results in VLPs that can deliver antibodies into mammalian cells [1]. As SeMV is amenable to genetic and chemical modifications, we have analysed the internal and external surface features of SeMV NPs with the goal of attaching dyes for bioimaging applications. SeMV NPs bioconjugated with fluorophores were characterized and tested for their ability to enter various types of mammalian cells by confocal microscopy and flow cytometry. The results demonstrate that although the virus can enter HeLa, HepG2, MDA-MB-231 and NIH/3T3 cells, it has preference for MDA-MB-231 cells. The interaction of VNPs with cell-surface proteins was demonstrated using far-western blot and mass spectroscopic analyses. The preferential binding property of SeMV NPs can be exploited for using them as sensors for intravital imaging and as nanocarriers for targeted drug delivery. To the best of our knowledge, this is the first report on the utilization of plant VNPs for imaging from India.

\section{Materials and methods}

\section{Bioconjugation of fluorophores to SeMV NPs}

SeMV was propagated on $S$. grandiflora plants maintained in a greenhouse and purified by ultracentrifugation as described earlier [12]. The surface-exposed lysine residue on SeMV was conjugated to Alexa Fluor 488 NHS ester (A488; Thermo), whereas the cysteine residues were conjugated to cyanine 5.5 maleimide (Cy5.5; Lumiprobe). Prior to Cy5.5 labelling, SeMV was treated with a 1000fold molar excess of dithiothreitol (DTT) to reduce the disulfide bond between $\mathrm{C} 169$ and C176. Excess DTT was removed using $10-\mathrm{kDa}$ centrifugal spin filters. The fluorophores (540 molar excess of A488 for external labelling and 900 molar excess of Cy5.5 for internal labelling) were added to SeMV $(1 \mathrm{mg} / \mathrm{ml})$ in $10 \mathrm{mM}$ potassium phosphate buffer, pH 7.0 (KP buffer), in the presence of 10\% DMSO. The reaction was carried out for $2 \mathrm{~h}$ (A488) or overnight (Cy5.5) at room temperature with constant stirring in the dark. SeMV NPs from both reaction mixtures were purified through a $30 \%(\mathrm{w} / \mathrm{v})$ sucrose cushion by ultracentrifugation at 48,000 rpm for $2 \mathrm{~h}$ using an MLS 50 rotor (Beckman Coulter Inc.). Pellets containing the dyelabelled particles were resuspended in KP buffer and left overnight at $4{ }^{\circ} \mathrm{C}$. Aggregates, if any, were removed by spinning at $12,000 \mathrm{rpm}$ for $10 \mathrm{~min}$.

\section{UV-Visible spectroscopy}

A NanoDrop 2000 spectrophotometer (Thermo Scientific) was used to characterize the UV-Vis spectra of native and modified SeMV NPs. The fluorophore load was determined using the protein concentration estimated by the Bradford method and the specific extinction coefficients of the respective compounds: A488, $\varepsilon(494 \mathrm{~nm})=73,000 \mathrm{M}^{-1} \mathrm{~cm}^{-1}$; Cy5.5, $\varepsilon(673 \mathrm{~nm})=209,000 \mathrm{M}^{-1} \mathrm{~cm}^{-1}$. The following molecular masses were used: SeMV, $5.4 \times 10^{6} \mathrm{~g} \mathrm{~mol}^{-1}$; Alexa Fluor 488 NHS, $643.4 \mathrm{~g} \mathrm{~mol}^{-1}$; Cy5.5 maleimide, $741.36 \mathrm{~g} \mathrm{~mol}^{-1}$.

\section{Denaturing gel electrophoresis}

Native and modified SeMV NPs (10 $\mu$ g per lane) were analyzed by SDS-PAGE using $12 \%$ gels and Tris-glycine ( 0.025 M Tris, $0.192 \mathrm{M}$ glycine) buffer, $\mathrm{pH}$ 8.3. Gel images were captured under UV and white light before staining with Coomassie blue, and under white light using an Image Quant system (GE Healthcare) after staining.

\section{Size-exclusion chromatography (SEC)}

Native and modified SeMV NPs were analyzed by SEC using a Superose 6 10/300 GL column on an Akta Purifier system (GE Healthcare) by loading $100 \mu \mathrm{g}$ of the sample at a flow rate of $0.5 \mathrm{ml} / \mathrm{min}$. 


\section{Transmission electron microscopy (TEM)}

Ten $\mu \mathrm{l}$ of native and modified SeMV NPs $(0.1 \mathrm{mg} / \mathrm{ml})$ suspended in KP buffer was deposited onto Formvar carboncoated copper grids (SPI supplies) for $2 \mathrm{~min}$ at room temperature (RT) followed by washing twice with deionized water for $60 \mathrm{~s}$. The samples were negatively stained with $2 \%$ uranyl acetate (UAc) for $45 \mathrm{~s}$ and washed with deionized water for $30 \mathrm{~s}$. The samples were analyzed using a FEI Tecnai transmission electron microscope at $120 \mathrm{kV}$ with a magnification of $135,000 x$.

\section{Tissue culture}

HeLa (cervical cancer), HepG2 (hepatic cancer), MDAMB-231 (breast cancer) and NIH/3T3 (mouse fibroblast) cells were procured from the American Type Culture Collection (ATCC) and maintained in Dulbecco's modified Eagle's medium (DMEM) supplemented with 10\% (v/v) fetal bovine serum (Gibco), 1\% (w/v) penicillin/streptomycin (Thermo scientific) and $1 \%$ glutamine (Glutamax, Thermo Scientific) at $37{ }^{\circ} \mathrm{C}$ and $5 \% \mathrm{CO}_{2}$.

\section{Confocal microscopy}

The cell lines were grown overnight on glass cover slips $(25,000$ cells per well) placed in an untreated 12-well plate in $200 \mu \mathrm{l}$ of $10 \%$ DMEM. The cells were washed twice with Dulbecco's PBS (DPBS) to remove unbound and dead cells before addition of A488-SeMV or SeMV-Cy5.5 particles $\left(5 \times 10^{6}\right.$ particles per cell, corresponding to $\sim 1 \mu \mathrm{g} /$ well $)$ and incubated for 0.5, 2 and $6 \mathrm{~h}$. The cells were washed three times with DPBS to remove unbound particles and fixed with $4 \%$ paraformaldehyde in PBS for $5 \mathrm{~min}$ at RT. The nucleus was stained with DAPI $(10 \mathrm{ng} / \mathrm{ml})$ for $5 \mathrm{~min}$ at RT and washed twice with PBS. The coverslips were mounted on glass slides using Fluoroshield and sealed with nail polish. Confocal images were captured on a Zeiss Airyscan microscope, and the images were processed using the Zen software.

For live cell imaging, $2 \times 10^{5}$ MDA-MB-231 cells were seeded into a $35-\mathrm{mm}$ glass-bottomed Petri dish and incubated overnight. One $\mu \mathrm{g}$ of SeMV-Cy5.5 particles was added, and images were captured every $5 \mathrm{~min}$, while recording the events continuously using a Zeiss Airyscan microscope for a period of $75 \mathrm{~min}$.

\section{Fluorescence-activated cell sorting (FACS)}

$2 \times 10^{6}$ cells were grown overnight in a $12-$ well plate. A488SeMV and SeMV-Cy5.5 NPs (100,000 particles per cell, corresponding to $\sim 0.18 \mu \mathrm{g} / \mathrm{well}$ ) were added to the cells and incubated for $0.5,2$, and $6 \mathrm{~h}$. The cells were washed twice with PBS and trypsinized. The cells were collected carefully in a sterile 1.5-ml Eppendorf tube and fixed with 2\% paraformaldehyde for $10 \mathrm{~min}$ at RT. The cells were washed, resuspended in PBS, and analyzed using a BD FACS Verse flow cytometer. Triplicates of each sample were examined, and at least 10,000 events (gated for live cells) were recorded. Data were analyzed using the BD FACS Suite software.

\section{Far-western blot analysis}

Far-western blot analysis [23] was used to detect cell membrane proteins that interact with SeMV NPs. Membrane proteins were isolated from HeLa cells using an Mem-PER plus membrane protein extraction kit (Thermo Fisher). Briefly, 50 $\mu \mathrm{g}$ of a HeLa cell membrane protein extract was loaded onto a $12 \%$ SDS-PAGE gel, and after electrophoresis, the proteins were transferred to a PVDF membrane. The transferred proteins were renatured (DR buffer, Supplementary Table 1) by gradually reducing the guanidine- $\mathrm{HCl}$ concentration $(6,3,1$, $0.1,0 \mathrm{M}$ ) and blocked using 5\% skim milk. Following this, the membrane was incubated overnight with SeMV NPs (10 $\mu \mathrm{g} / \mathrm{ml}$ ) and washed thoroughly. Bound SeMV was detected using an SeMV polyclonal antiserum (1:5,000), followed by goat anti-rabbit HRP conjugated secondary antibody at a 1:10,000 dilution, and the interacting proteins were visualized using chemiluminescence.

\section{In-gel digestion and LC-MS/MS analysis}

The HeLa membrane protein bands that were visible in the far-western blot upon interaction with SeMV were excised from the SDS-PAGE gel using a sterile blade and transferred to $1.5-\mathrm{mL}$ tubes. The gel pieces were destained using 40 $\mathrm{mM}$ ammonium bicarbonate in $40 \%$ acetonitrile (ACN). The samples were then subjected to reduction using $5 \mathrm{mM}$ DTT $\left(60{ }^{\circ} \mathrm{C}\right.$ for $35 \mathrm{~min}$ ) followed by alkylation using $20 \mathrm{mM}$ iodoacetamide (10 min at RT). In-gel digestion with trypsin was carried out overnight at $37^{\circ} \mathrm{C}$ using MS grade trypsin (10 ng/ $\mu \mathrm{L}$ ) (Sigma Aldrich, USA). Peptides were extracted sequentially by treating the gel pieces with $5 \%$ formic acid, $5 \%$ formic acid in $40 \%$ ACN, and finally, $100 \%$ ACN. The extracted peptides were vacuum dried and used for LC-MS/ MS analysis. Samples were analyzed on a Thermo Scientific Orbitrap Fusion Tribrid Mass Spectrometer (Thermo Scientific, Bremen, Germany) interfaced with an Easy-nLC II nanoflow liquid chromatography system (Thermo Scientific, Odense, Denmark). Mascot and SEQUEST search algorithms were used for data analysis against the Human RefSeq75 protein database supplemented with frequently observed contaminants through the Proteome Discoverer platform (v1.4, Thermo Scientific, Bremen, Germany). The data were searched against a decoy database, and the results 
from both searches were used to estimate q-values using the Percolator algorithm within the Proteome Discoverer suite.

\section{Treatment of cell lines with anti-vimentin antibodies}

To examine the role of vimentin in the internalization of SeMV NPs, HeLa, HepG2, MDA-MB-231 and NIH/3T3 cells were grown to confluency and then seeded in a 6 -well plate at $2 \times 10^{5}$ cells/well and incubated overnight. The cells were treated with $10 \mathrm{ng}$ of anti-vimentin antibodies per $\mu \mathrm{l}$ for $2 \mathrm{~h}$. The medium was then removed, and fresh medium was added to the cells. Two $\mu \mathrm{g}$ of SeMV-Cy5.5 was added to the cells, which were then incubated for $6 \mathrm{~h}$. The medium was then removed, and the cells were washed twice with PBS. The cells were trypsinized and pelleted at $3000 \mathrm{rpm}$ for 2 min. The cell pellet was washed twice with PBS and fixed in $2 \%$ paraformaldehyde for $30 \mathrm{~min}$. The cells were washed twice with PBS and processed for FACS analysis.

\section{Results}

\section{Characterization of native SeMV NPs}

The yield of SeMV purified from infected sesbania leaves was $1 \mathrm{~g}$ per $\mathrm{kg}$ of leaf material as determined using the Bradford assay. Ultracentifugation using a 10-40\% (w/v) linear sucrose density gradient revealed a single light-scattering zone (Fig. S1A). The recovered zone fraction eluted as a single peak at $8 \mathrm{ml}$ in size exclusion chromatography (SEC), suggesting that the purified fraction corresponded to intact assembled particles (Fig. S1B). SDS-PAGE analysis of a denatured SeMV preparation revealed a single 30-kDa band corresponding to the molecular weight of the coat protein (Fig. S1C). Transmission electron microscopy (TEM) revealed that the SeMV NPs were spherical in shape and 30 $\pm 1 \mathrm{~nm}$ in diameter (Fig. S1D).
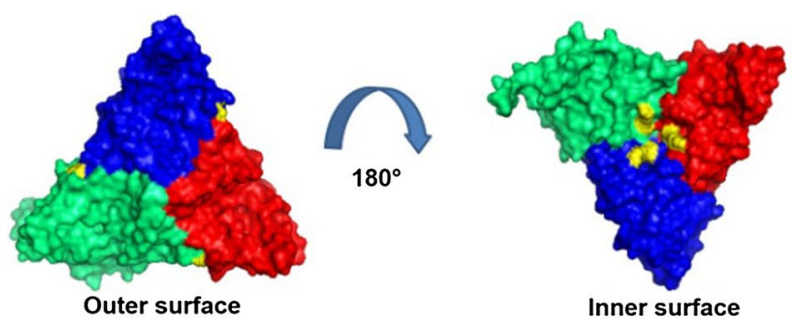

Fig. 1 Structure of SeMV (PDB ID: 1SMV) showing the probable sites of bioconjugation. The $\mathrm{A}, \mathrm{B}$ and $\mathrm{C}$ subunits of the icosahedral asymmetric unit of SeMV are shown in blue, red, and green, respec-

\section{Location of sites in the SeMV structure suitable for attaching dyes}

Design of an SeMV-based platform for biomedical applications requires identification of sites on the capsid that are suitable for bioconjugation reactions and do not compromise the structure and assembly of the virus. The crystal structure of SeMV (PDB: 1SMV) was examined to locate such sites on the exterior and interior surfaces of the capsid. Of the five lysine residues of the SeMV CP, one (K149) is exposed on the exterior surface, resulting in 180 lysine residues per VNP that can be used for N-hydroxysuccinimide (NHS)-ester-based bioconjugation (Fig. 1). Four cysteine residues $(\mathrm{C} 72, \mathrm{C} 123, \mathrm{C} 169, \mathrm{C} 176$; the latter two involved in a disulfide bond) of the $\mathrm{CP}$ that are buried constitute 720 sites per VNP that are available for thiol-maleimide-based bioconjugation (Fig. 1).

\section{Bioconjugation of SeMV NPs with fluorophores}

The lysine residues of SeMV were conjugated to NHS-activated esters of A488 (540-fold molar excess equivalent to 3 dye molecules per $\mathrm{CP}$ subunit) for $2 \mathrm{~h}$. Internal cysteine residues of SeMV were conjugated by overnight incubation to maleimide esters of Cy5.5 (900-fold molar excess equivalent to 5 dye molecules per $\mathrm{CP}$ subunit). The resulting dye-labelled SeMV particles (referred to as A488-SeMV and SeMV-Cy5.5) were purified by ultracentrifugation to remove excess free dye. A Bradford assay was performed to estimate the protein concentration, and UV-visible spectroscopy was used to determine the number of dye molecules per particle based on the extinction coefficient of the dye. It was estimated that A488-SeMV NPs contained 40-50 molecules of A488 and SeMV-Cy5.5 contained 420-440 molecules of Cy5.5.

A488-SeMV and SeMV-Cy5.5 were examined by SDSPAGE, size exclusion chromatography and TEM. Visualization of the SDS-PAGE gel under UV light for A488-SeMV (Fig. 2A, lane 2) and under white light for SeMV-Cy5.5 (Fig. 2B, lane 3) before Coomassie blue staining confirmed
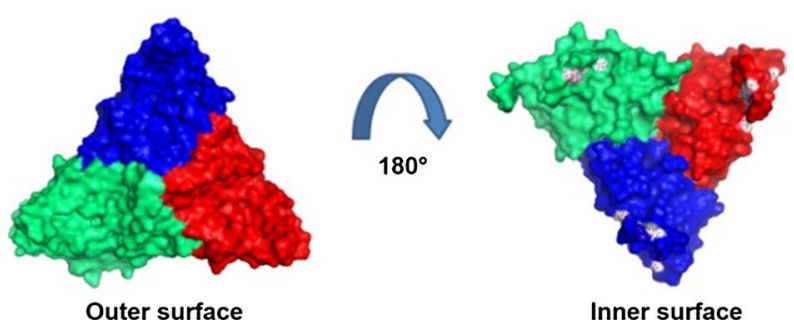

tively. The external (K149) and internal (K109, K194, K195, K200) lysines and cysteines (C72, C123, C169, C172) are shown in yellow and white, respectively. The images were created using PyMol 
the covalent attachment of the dyes. Dye-labelled particles eluted as a single peak at the same elution volume as that of native SeMV $(8 \mathrm{ml})$ when passed through a Superose-6 column indicating that they were intact. The fluorophore was co-eluted with A488-SeMV and SeMV-Cy5.5 particles with the expected absorbance at 495 and $673 \mathrm{~nm}$, respectively (Fig. 2C). TEM analysis showed that the labelled particles remained monodisperse with a diameter of $30 \pm$
A

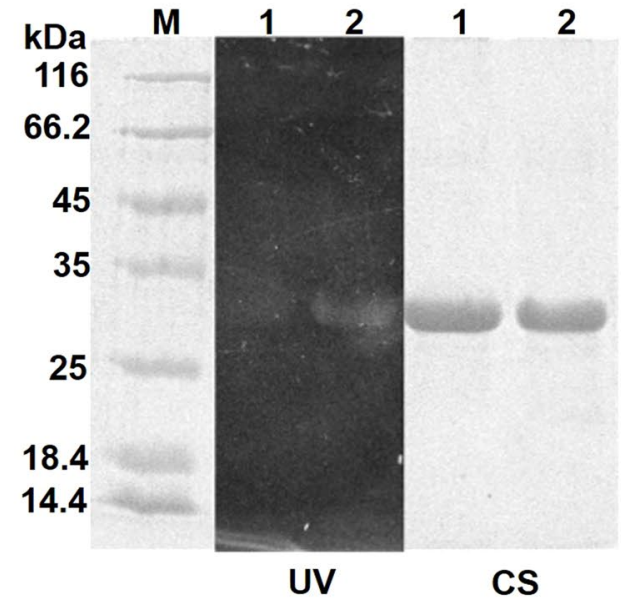

C

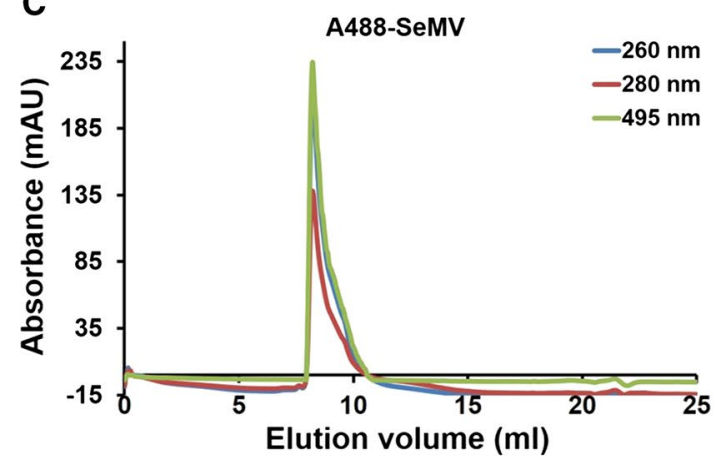

D

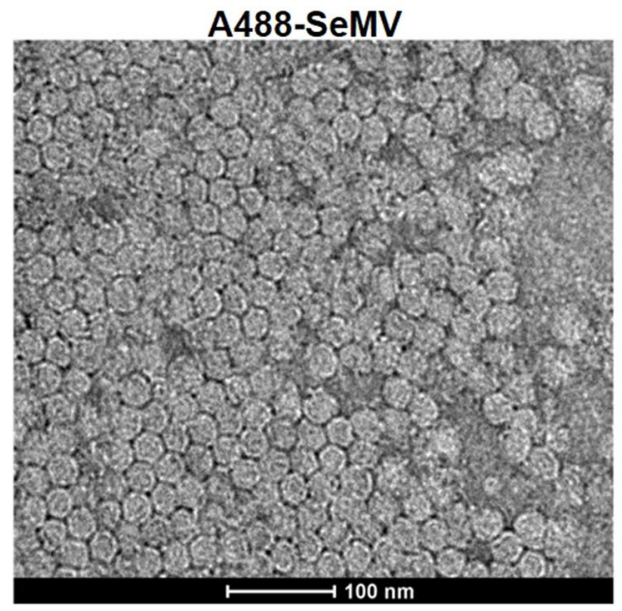

Fig. 2 Characterization of fluorophore-labelled SeMV NPs. SDSPAGE analysis of A) A488-SeMV and B) SeMV-Cy5.5 visualized under UV light (UV) and white light (WL), respectively, before staining and under white light after Coomassie brilliant blue staining (CS). M), Fermentas unstained protein ladder; 1, SeMV NPs; 2,
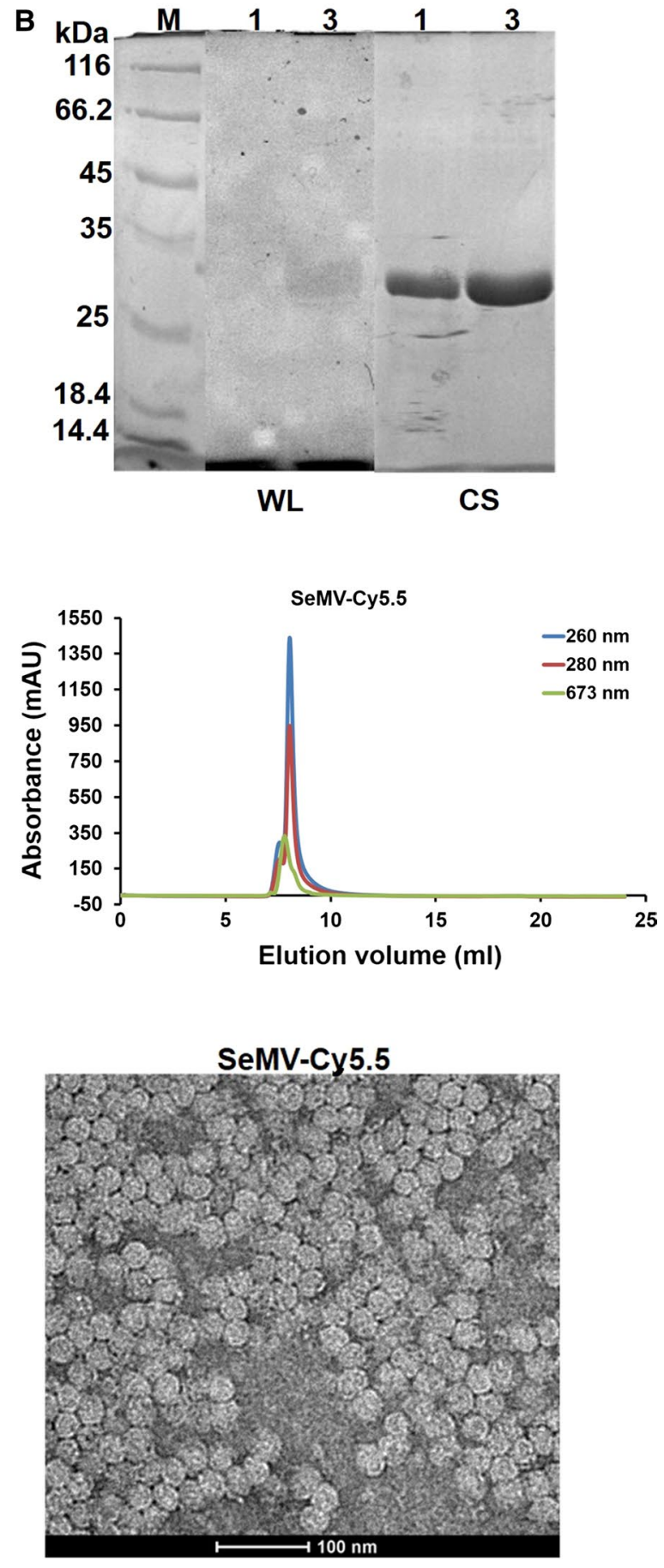

A488-SeMV; 3, SeMV-Cy5.5. C) Size exclusion chromatograms of A488-SeMV (monitored at $260 \mathrm{~nm}$ [blue], $280 \mathrm{~nm}$ [red] and $495 \mathrm{~nm}$ [green]), SeMV-Cy5.5 (monitored at $260 \mathrm{~nm}$ [blue], $280 \mathrm{~nm}$ [red], and $673 \mathrm{~nm}$ [green]). D) Transmission electron micrographs of uranyl-acetate-stained A488-SeMV and SeMV-Cy5.5 
$2 \mathrm{~nm}$ based on ImageJ analysis (Fig. 2D). When stored at 4 ${ }^{\circ} \mathrm{C}$ in KP buffer for 3 months, the particles continued to be stable. However, the fluorescence intensity of A488-SeMV was completely lost, and there was a $40 \%$ reduction in the fluorescence intensity of SeMV-Cy5.5, suggesting that the fluorescence of the latter particles is much more stable (Fig. $\mathrm{S} 2 \mathrm{~A}$ and $\mathrm{B}$ ).

\section{Imaging studies}

Cervical cancer (HeLa), hepatic cancer (HepG2), breast cancer (MDA-MB-231) and mouse fibroblast (NIH/3T3) cells were incubated with A488-SeMV and SeMV-Cy5.5 particles separately for $0.5,2$ and $6 \mathrm{~h}$ before confocal microscopy and FACS analysis (Fig. 3 A, B and D). Although SeMV replication is restricted to plants, based on the fluorescence intensity, it was observed that A488-SeMV and SeMV-Cy5.5 particles were internalized into all four mammalian cell lines tested (Fig. 3A and B). A488-SeMV particles showed a tenfold lower intensity than SeMV-Cy5.5 particles when measured at the same concentration (data not shown). It was observed in the FACS analysis that the mean fluorescence intensity (MFI) increased for $6 \mathrm{~h}$ after incubation of different mammalian cell lines with SeMV-Cy5.5. Interestingly, the MFI of MDA-MB-231 cells incubated with SeMV-Cy5.5 NPs were 3.9-fold higher at the 6-h time point and 2.2- and 1.8-fold higher in HepG2 and NIH/3T3 cells, respectively, than that in HeLa cells suggesting that the kinetics of SeMVCy5.5 entry may vary among different cell lines (Fig. 3D). Indeed, live-cell imaging experiments with MDA-MB-231 cells showed that SeMV-Cy5.5 can bind to cells in $5 \mathrm{~min}$, and entry was observed within 15 min (Fig. 3C). Confocal imaging showed colocalization of SeMV-Cy5.5 with the endolysosomal marker LAMP-1 at $6 \mathrm{~h}$ in all four cell lines tested, suggesting that SeMV-Cy5.5 undergoes endocytosis and is degraded via the lysosomal pathway (Fig. S3).

\section{Identification of cell membrane proteins interacting with SeMV}

Far-western blot analysis was used to identify the cell a membrane proteins of HeLa cells that interact with SeMV. Three proteins corresponding to molecular mass of 54, 35 and $33 \mathrm{kDa}$ were found prominently in the far-western blot (Fig. 4A). No band was seen when the blot was developed without preincubation with SeMV (data not shown), confirming that these proteins interact specifically with SeMV. The respective bands were excised from the gel and analyzed by mass spectrometry. Mass spectrometric analysis revealed that the 54-kDa protein band contained 32 peptides with masses that accounted for $70.4 \%$ of the polypeptide corresponding to vimentin, two other proteins of size $35 \mathrm{kDa}$ and $33 \mathrm{kDa}$, which corresponded to the voltage-dependent anion-selective channel protein (VDAC 1), identified with a coverage of $52.7 \%$ (11 peptides), and annexin A2 isoform 2 (ANXA2), identified with a coverage of $55.16 \%$ (16 peptides), respectively (Fig. 4B).

\section{Role of vimentin in internalization of SeMV NPs}

FACS analysis revealed that upon incubation of different cell lines with anti-vimentin antibodies, there was a significant decrease in the fluorescence intensity compared to the cells treated with SeMV-Cy5.5 alone. There was a 50\% reduction in the fluorescence intensity in MDA-MB-231 cells, followed by a $35 \%$ reduction in HepG2 and NIH/3T3 cells and a 27\% reduction in HeLa cells (Fig. 5). The results show that vimentin acts as one of the receptors for SeMV NPs for internalization into the cells and blocking the vimentin leads to significant decrease in the entry of SeMV NPs.

\section{Discussion}

Nanobiotechnology emphasizes the use of biologically derived structures smaller than $100 \mathrm{~nm}$, which can be engineered to execute specific functions. An ideal nanobiotechnological system should be able to produce particles of consistent size, structure, and biophysical properties and should tolerate the introduction of other functional groups such as fluorophores, peptides, or inorganic compounds. Plant VNPs possess all of the attributes required for a particle to be tailored as a potential nanocarrier for multiple applications. SeMV is a well-characterized plant virus that was chosen for the current work. As described in the results section, SeMV forms homogeneous particles of $30 \mathrm{~nm}$ in diameter hat are quite stable. The crystal structure of SeMV has been well studied, and amenable sites for bioconjugation of fluorophores were identified (Fig. 1). SeMV was conjugated with Alexa Fluor 488 externally and Cy 5.5 internally, respectively. The labelled particles were found to be structurally stable when analysed using SDS-PAGE, FPLC and electron microscopy (Fig. 2), indicating that the particles were even intact after being subjected to processes such as ultracentrifugation. Fluorescence data showed that the A488-SeMV signal was low compared to that of SeMV-Cy 5.5 (Fig. 3A, B, and $\mathrm{D})$. This contrasts with the results for the exterior- and interior-labelled CPMV particles, where the signal obtained from internally labelled CPMV was lower than that from externally labelled particles. Native SeMV has a zeta potential of $-18.1 \mathrm{mV}$ (Supplementary Table 2), while CPMV has $-7.5 \mathrm{mV}$ [10]. Usually, nanoparticles with a zeta potential higher than $+25 \mathrm{mV}$ and lower than $-25 \mathrm{mV}$ display a high degree of stability. This may be the reason for the increased stability of the labelled fluor in SeMV-Cy5.5 (-34 mV) compared to A488-SeMV (-11.6 mV) and internally labelled 
Fig. 3 Cellular internalization studies with fluorescent colabelled SeMV NPs analyzed by confocal fluorescent microscopy and FACS. Confocal images showing the time dependent internalization of A) A488SeMV (green) and B) SeMVCy5.5 (red) in HeLa, HepG2, MDA-MB-231 and NIH/3T3 cells. C) Live cell imaging panels of MDA-MB-231 cells incubated with SeMV-Cy5.5 for a period of $75 \mathrm{~min}$. The panels represent images captured at 5-min intervals. D) FACS analysis of HeLa, HepG2, MDAMB-231 and NIH/3T3 cells incubated with SeMV-Cy5.5 for $0.5,2$ and $6 \mathrm{~h}$. Error bars represent the standard deviation of the mean for the samples
A
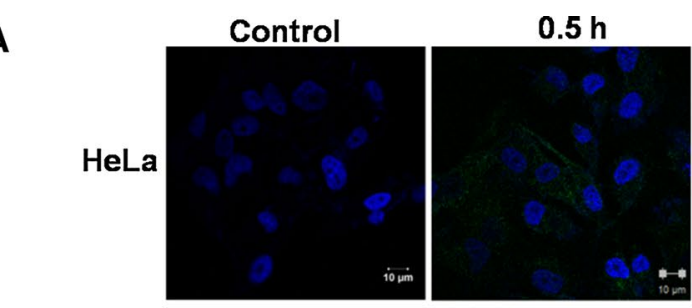

$2 \mathrm{~h}$

$6 \mathrm{~h}$

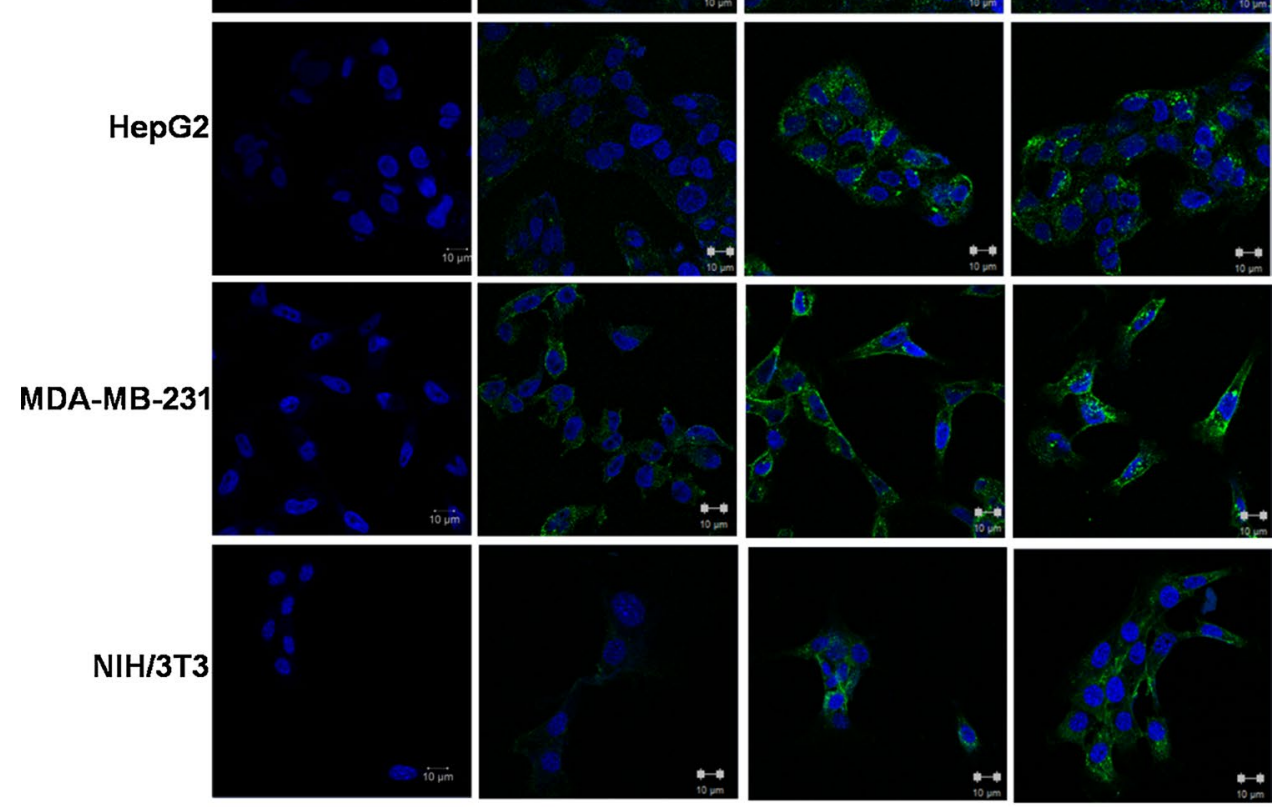

B

Control

$0.5 \mathrm{~h}$

$2 \mathrm{~h}$

$6 \mathrm{~h}$

MDA-MB-231

io 0 mim

HepG2

NIH/3T3
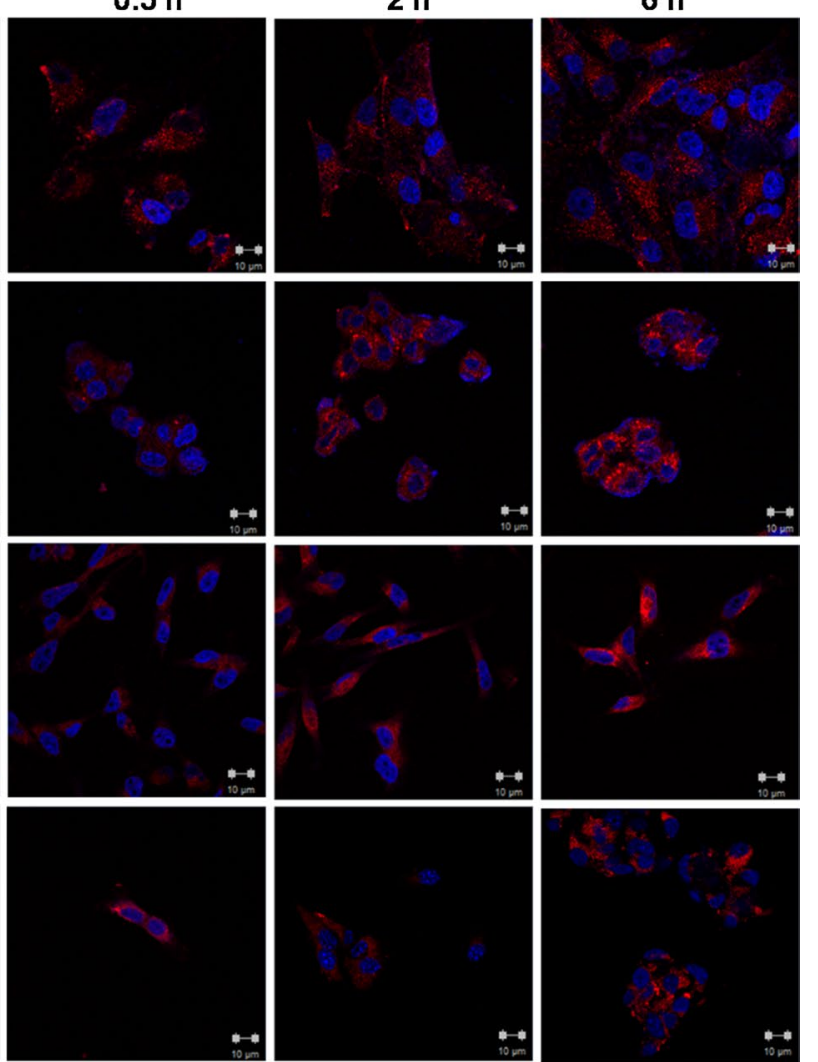
Fig. 3 (continued)

\section{C}

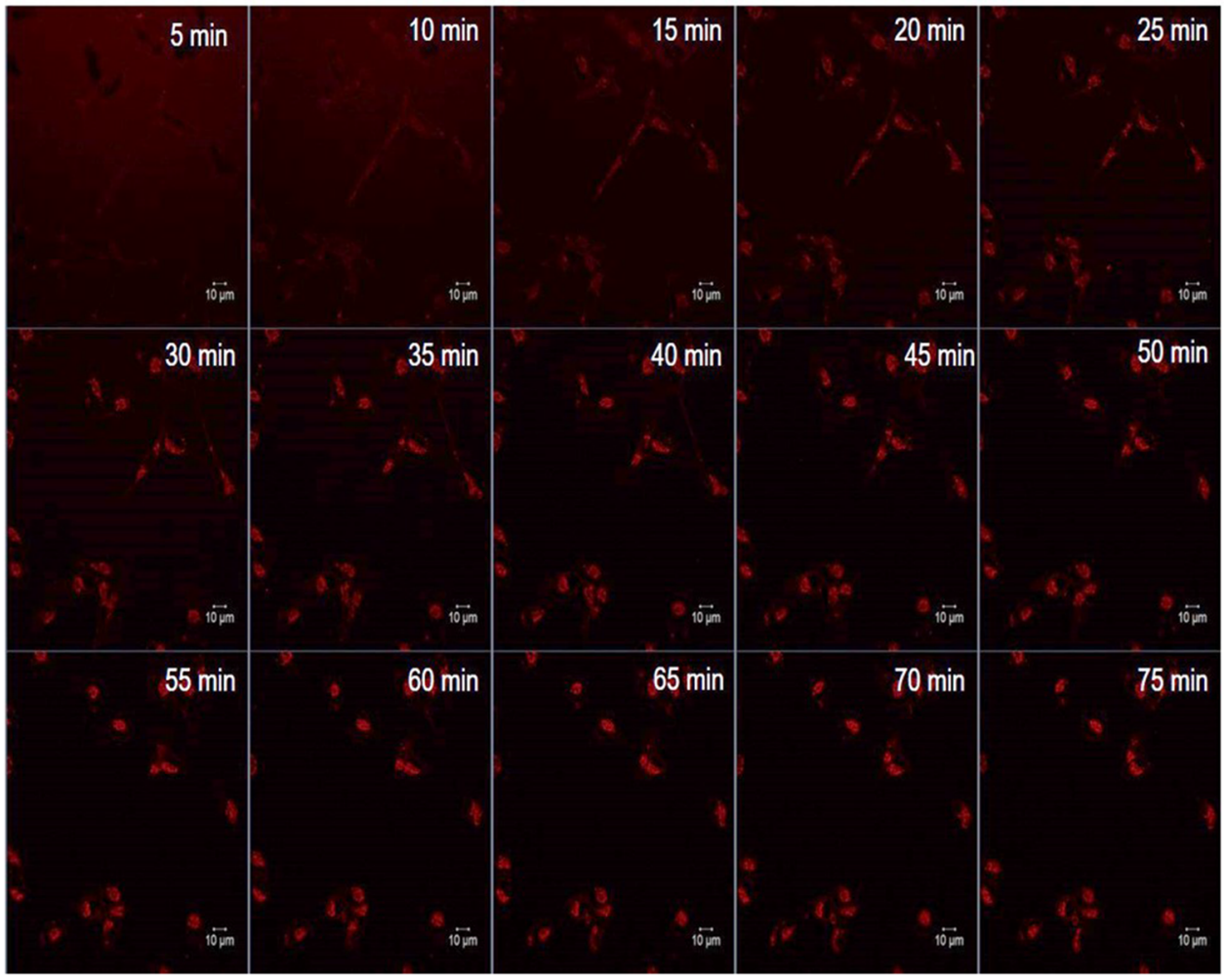

D

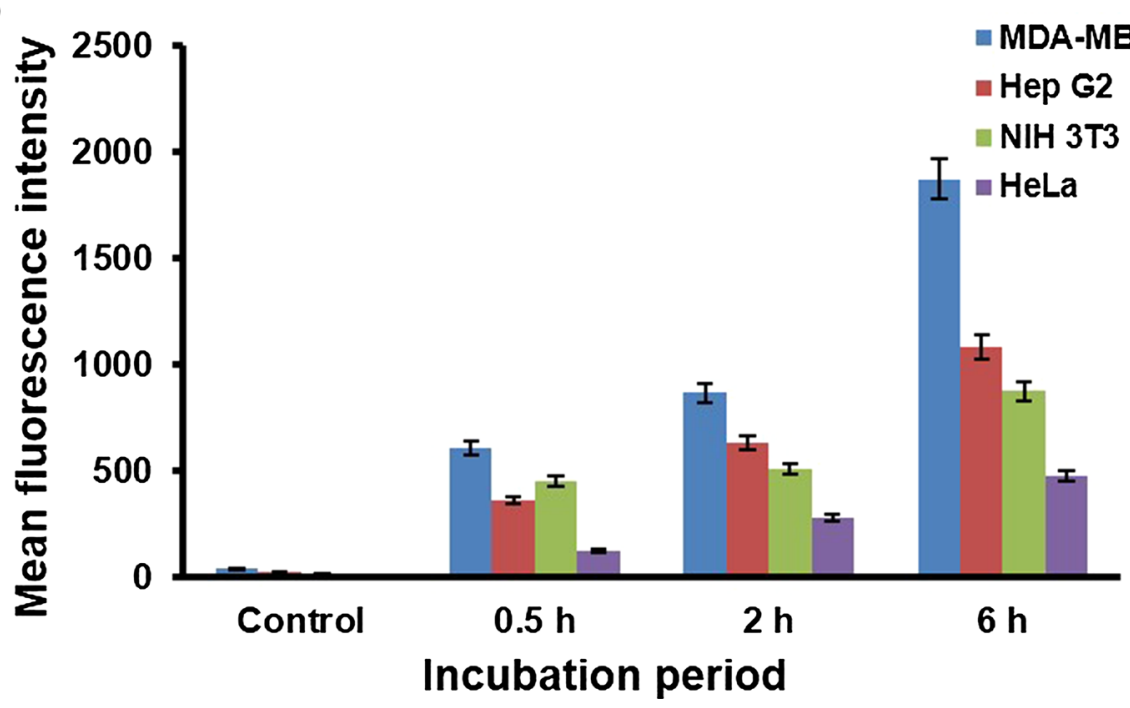

CPMV. SeMV is similar to CPMV in terms of size, shape, and charge (negative), but they are different with respect to the number of capsid subunits and subunit arrangement in the $\mathrm{T}=3$ icosahedral structure. Moreover, the differences in the surface chemistry available for functionalization could contribute to differences in cell uptake of the labelled particles [12]. On the other hand, internally labelled PhMV VLPS were shown to be more sensitive and stable than externally labelled particles, although the zeta potential of the former has been reported to be $-7.92 \mathrm{mV}$ [9]. Colocalization of SeMV-Cy5.5 with LAMP-1 antibodies revealed that SeMV NPs undergo degradation via the lysosomal pathway (Fig. S3). It was reported earlier that CPMV [18] and PhMV [9] also undergo degradation via the lysosomal pathway.

It is evident from the far-western blot analysis that SeMV NPs interact predominantly with vimentin, VDAC 1 and ANXA2 from the HeLa cell membrane protein fraction (Fig. 4A). It has been demonstrated that the interaction 
Fig. 4 Identification of cell membrane proteins interacting with SeMV. A) Far-western blot of a HeLa cell membrane protein fraction treated with SeMV, showing its interaction with proteins corresponding to a molecular weight of 54,35 and $33 \mathrm{kDa}$. M, Prestained protein marker (Bluestar, Genetics); 1, HeLa membrane protein fraction. B) Mass spectrometry data of 54-kDa, 35-kDa and 33-kDa protein bands that interact with SeMV, revealing their identity as vimentin, voltage-dependent anion protein channel, and annexin A2 isoform 2, respectively

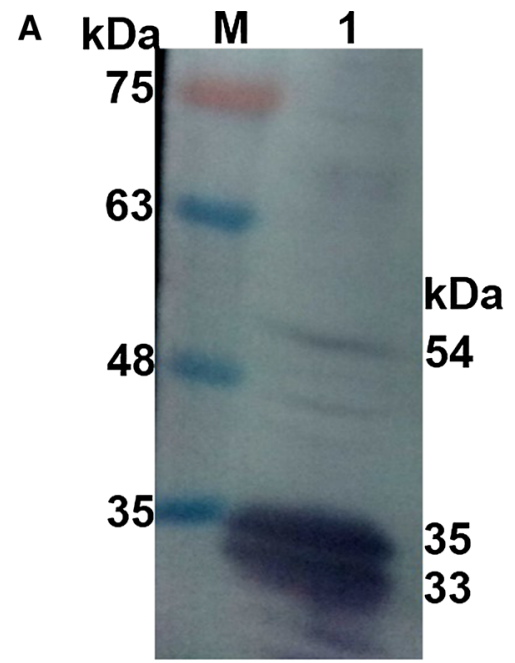

B

\begin{tabular}{|c|c|c|c|c|}
\hline $\begin{array}{c}\text { MW of } \\
\text { band } \\
\text { analyzed }\end{array}$ & Protein Description & $\begin{array}{c}\% \\
\text { Coverage }\end{array}$ & $\begin{array}{c}\text { No. of } \\
\text { Peptides }\end{array}$ & $\begin{array}{c}\text { Theoretical } \\
\text { MW [kDa] }\end{array}$ \\
\hline $54 \mathrm{kDa}$ & $\begin{array}{c}\text { Vimentin [Homo } \\
\text { sapiens] }\end{array}$ & 70.39 & 41 & 53.6 \\
\hline $35 \mathrm{kDa}$ & $\begin{array}{c}\text { Voltage-dependent } \\
\text { anion-selective } \\
\text { channel protein 1 } \\
\text { [Homo sapiens] }\end{array}$ & 52.65 & 11 & 30.8 \\
\hline $33 \mathrm{kDa}$ & $\begin{array}{c}\text { Annexin A2 isoform } \\
\text { 2 [Homo sapiens] }\end{array}$ & 55.16 & 16 & 38.6 \\
\hline
\end{tabular}

Fig. 5 Effect of anti-vimentin antibodies on the internalization of SeMV NPs. A significant decrease in the fluorescence intensity in flow cytometry analysis was observed in all of the cell lines (MDA-MB-231, $50 \%$; HepG2, 35\%; NIH/3T3, $35 \%$; HeLa, 27\%) treated with anti-vimentin antibodies, indicating the role of vimentin as one of the routes of internalization. Error bars represent the standard deviation of the mean for the samples

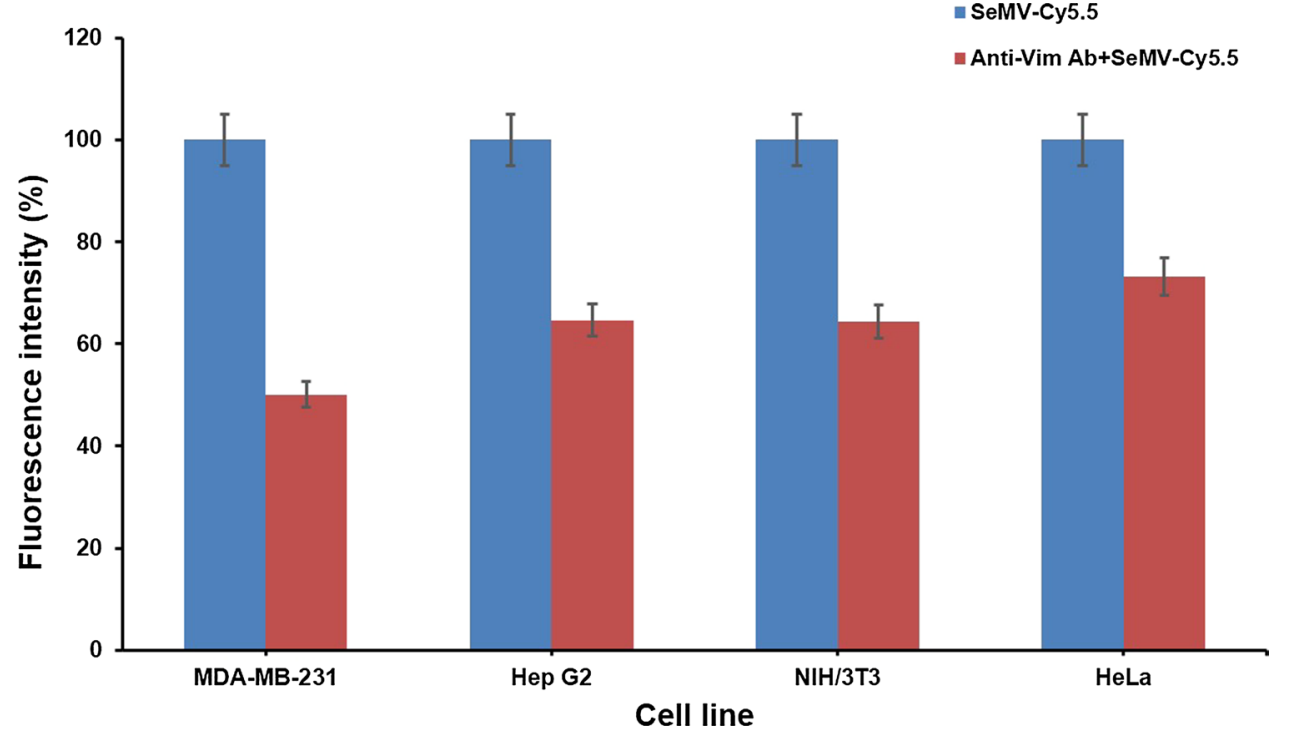


of CPMV with mammalian cells is mediated by surfaceexposed vimentin. Vimentin is a cytoskeletal protein that controls the architecture and dynamics of the interior of cells. Several studies have demonstrated the use of surface vimentin as an attachment receptor by bacterial and viral pathogens. Studies of CPMV-vimentin interactions have demonstrated that increased surface vimentin is a significant feature of tumor endothelium which might indicate a role of surface vimentin in tumor metastasis besides cellular adhesion and stress [10]. CPMV uses caveolar endocytosis and macropinocytosis pathways for cellular internalization [18]. It is possible that SeMV NPs are also internalized into mammalian cells through vimentin and follow the same degradation pathway as that of CPMV. Although maximum coverage (70\%) was obtained with vimentin, a few other proteins with greater than $40 \%$ coverage (Supplementary Table 3) were also identified. However, none of these other proteins are known to be receptors, and they are therefore not relevant. Blocking studies with vimentin-specific antibodies showed that vimentin is indeed one of the binding partners for SeMV internalization (Fig. 5)

Unlike CPMV, SeMV NPs were found to interact with two other proteins of $35 \mathrm{kDa}$ and $33 \mathrm{kDa}$ in size, which corresponded to VDAC-1 and ANXA2, respectively (Fig. 4B). However, the calculated masses of VDAC-1 (30.8 kDa) and ANXA2 (38.6 kDa) are lower (35 kDa) and higher (33 $\mathrm{kDa}$ ), respectively than the observed masses, and this therefore needs further authentication. VDAC-1 forms a channel through the mitochondrial outer membrane and the plasma membrane. The channel in the plasma membrane is involved in cell volume regulation and apoptosis [5]. Annexins are a family of proteins that bind to anionic phospholipids in a calcium-dependent manner. Like other members of the family, ANXA2 is a pleiotropic protein and is involved in diverse cellular processes such as cell motility, endocytosis, fibrinolysis, ion channel formation, and cell matrix interaction [25]. However, there are no reports on the role of VDAC1 and ANXA2 in the internalization of plant virus particles into mammalian cells. Based on the far-western blot and mass spectrometry data, it can be hypothesized that SeMV interacts with multiple proteins and perhaps utilizes different pathways for being internalized into different cells.

SeMV NPs are non-infectious and non-toxic to humans and are biodegradable. Their stability upon functionalization and easy, inexpensive and scalable production makes them excellent candidates for biomedical applications. These particles were shown to have a natural preference for certain cells, which could be exploited further for targeted delivery of drugs and for in vivo imaging for future use in cancer diagnosis and therapy.

Acknowledgements This work was funded by Department of Biotechnology, India (BT/PR6711/NNT/28/622/2012). We also thank the
Indian Institute of Science for financial support and for all the facilities. The Institute of Bioinformatics, Bengaluru, is gratefully acknowledged for help with mass spectrometric analysis. MRN and HSS thank the Department of Science and Technology (DST) for a J.C. Bose fellowship, and the Indian National Science Academy (INSA) for financial support. Vishnu Vardhan acknowledges DST-INSPIRE for providing a fellowship.

\section{References}

1. Abraham A, Nataraj U, Karande AA, Gulati A, Murthy MRN, Murugesan S, Mukunda P, Savithri HS (2016) Intracellular delivery of antibodies by chimeric Sesbania mosaic virus (SeMV) virus like particles. Sci Rep 6:21803. https://doi.org/10.1038/srep21803

2. Gulati A, Murthy A, Abraham A, Mohan K, Natraj U, Savithri HS, Murthy MRN (2016) Structural studies on chimeric Sesbania mosaic virus coat protein: revisiting SeMV assembly. Virology 489:34-43

3. Bakshi A, Vishnu Vardhan GP, Hema M, Murthy MRN, Savithri HS (2017) Structural and functional characterization of sesbania mosaic virus. In: Mandal B, Rao G, Baranwal V, Jain R (eds) A century of plant virology in India. Springer, Singapore, pp 405-427

4. Bhuvaneshwari M, Subramanya HS, Gopinath K, Savithri HS, Nayudu MV, Murthy MRN (1995) Structure of Sesbania mosaic virus at $3 \AA$ Å resolution. Structure 3:1021-1030

5. Chang JS, Chi SC (2015) GHSC70 is involved in the cellular entry of nervous necrosis virus. J Virol 89:61-70

6. Culver JN, Brown AD, Zang F, Gnerlich M, Gerasopoulos K, Ghodssi R (2015) Plant virus directed fabrication of nanoscale materials and devices. Virology 480:200-212

7. Czapar AE, Steinmetz NF (2017) Plant viruses and bacteriophages for drug delivery in medicine and biotechnology. Curr Opin Chem Biol 38:108-116

8. Govind K, Makinen K, Savithri HS (2012) Sesbania mosaic virus (SeMV) infectious clone: possible mechanism of $3^{\prime}$ and $5^{\prime}$ end repair and role of polyprotein processing in viral replication. PLoS One 7:e31190

9. Hema M, Bindi KP, Paul LC, He H, Neetu MG, Bradley LC, Reza AG, Sourabh S, Steinmetz NF (2017) Physalis mottle virus-like particles as nanocarriers for imaging reagents and drugs. Biomacromolecules 18:4141-4153

10. Koudelka KJ, Destito G, Plummer EM, Trauger SA, Siuzdak G, Manchester M (2009) Endothelial targeting of cowpea mosaic virus (CPMV) via surface vimentin. PLoS Pathog 5:e1000417

11. Koudelka KJ, Pitek AS, Manchester M, Steinmetz NF (2015) Virus-based nanoparticles as versatile nanomachines. Annu Rev Virol 2:379-401

12. Lee KL, Hubbard LC, Hern S, Yildiz I, Gratzl M, Steinmetz NF (2013) Shape matters: the diffusion rates of TMV rods and CPMV icosahedrons in a spheroid model of extracellular matrix are distinct. Biomater Sci 1:10

13. Lokesh GL, Gopinath K, Sathesh Kumar PS, Savithri HS (2001) Complete nucleotide sequence of Sesbania mosaic virus: a new virus species of the genus Sobemovirus. Arch Virol 146:209-223

14. Ma Y, Nolte RJ, Cornelissen JJ (2012) Virus-based nanocarriers for drug delivery. Adv Drug Deliv Rev 64:811-825

15. Mateu MG (2011) Virus engineering: functionalization and stabilization. Protein Eng Des Sel 24:53-63

16. Narayanan KB, Han SS (2017) Icosahedral plant viral nanoparticles-bioinspired synthesis of nanomaterials/nanostructures. Adv Colloid Interface Sci 248:1-19 
17. Narayanan KB, Han SS (2017) Helical plant viral nanoparticlesbioinspired synthesis of nanomaterials and nanostructures. Bioinspir Biomim 12:031001

18. Plummer EM, Manchester M (2013) Endocytic uptake pathways utilized by CPMV nanoparticles. Mol Pharm 10:26-32

19. Pokorski JK, Steinmetz NF (2011) The art of engineering viral nanoparticles. Mol Pharm 8:29-43

20. Sathesh Kumar PS, Lokesh GL, Murthy MRN, Savithri HS (2005) The role of arginine-rich motif and $\beta$-annulus in the assembly and stability of Sesbania mosaic virus capsids. J Mol Biol 35:3447-3458

21. Vishnu Vardhan GP, Savithri HS, Murthy MRN, Hema M (2016) Biodistribution and toxicity evaluation of Sesbania mosaic virus nanoparticles in mice. Arch Virol 161:2673-2681
22. Wen AM, Cho CF, Lewis JD, Steinmetz NF (2015) The application of plant viral nanoparticles in tissue-specific imaging. In: Mikhail YB (ed) Nanotechnology for biomedical imaging and diagnostics: from nanoparticle design to clinical applications. Wiley, New York, pp 401-427

23. Wu Y, Li Q, Chen Z (2007) Detecting protein-protein interactions by far western blotting. Nat Protoc 2:3278-3284

24. Young M, Willits D, Uchida M, Douglas T (2008) Plant viruses as biotemplates for materials and their use in nanotechnology. Annu Rev Phytopathol 46:361-384

25. Zhang S, Yu M, Guo Q, Li R, Li G, Tan S, Li X, Wei Y, Wu M (2015) Annexin A2 binds to endosomes and negatively regulated TLR4-triggered inflammatory responses via the TRAM-TRIF pathway. Sci Rep 5:15859 